\title{
Molecular Modeling, Dynamics Simulation and Characterization of Human Inositol Hexakisphosphate Kinase 1 (IP6K1) Related to Diabetes
}

\section{*1ELEKOFEHINTI, OO; ${ }^{2,4}$ ALADENIKA, YV; ${ }^{4}$ ALLI-SMITH, YR; , ${ }^{3}$ EJELONU, OC; ${ }^{1}$ LAWAL, AO}

\begin{abstract}
${ }^{1}$ Bioinformatics and Molecular Biology Unit, Department of Biochemistry, Federal University of Technology Akure, Ondo State, Nigeria, ${ }^{2,4}$ Biochemistry Unit, Science Laboratory Department, Gateway Polytechnic, Saapade, Ogun State ${ }^{3}$ Department of Biochemistry, Ondo State University of Science and Technology Okitipupa, Ondo State, Nigeria. ${ }^{4}$ Department of Biochemistry, University of Ado-Ekiti, AdoEkiti, Ekiti State, Nigeria
\end{abstract}

*Corresponding Author Email: sola_eleko@yahoo.com; ooelekofehinti@futa.edu.ng; Tel: +2348034450611

\begin{abstract}
Inositol hexakisphosphate kinase-1 (IP6K1) protein plays an important role in insulin signaling producing IP7 that inhibit the action of protein kinase B (Akt). Inhibition of IP6K1 has been proposed as a novel way to enhance insulin signaling. Characterization and binding interaction of IP6K1 is essential for rational anti-diabetic drug development targeting this protein. Computational tools were used to analyze the physicochemical characteristics of IP6K1. Homology modelling reliably predicts the tertiary structure of IP6K1. Derived three-dimensional models were then used to predict the binding mode and interacting amino acid residues. MD simulation (30 ns) was employed to investigate the protein dynamics. The modeled IP6K1 exhibited secondary characteristics comprising of $63.3 \%$ helixes, $30.2 \%$ sheets and $13.4 \%$ turns with an aliphatic index of 65.83 and instability index 50.53 showing that the protein is relatively unstable without its appropriate environment. The extinction coefficient was 34560 while the grand average of hydropathicity was -0.724 . Homology modelling was performed by SWISS-MODEL program and the proposed model was evaluated as reliable based on RAMPAGE's Ramachandran plot, and ProSA analyses. RMSD, RMSF, Rg revealed that the protein attained stability around 20ns. This appeared to be the first attempt to portray molecular dynamic simulation of IP6K1 coupled with modeling and thorough characteristic analysis of the protein using parameters like Ramachandran plot, Chou and Fasman Secondary Structure prediction and Protparam. Studies like protein engineering, structure and function as well as activity analysis are suggested. Our computational studies reavealed the binding pocket and critical amino acid residues that can be exploited in the design of inhibitors of IP6K1 as antidiabetic drugs.
\end{abstract}

\section{DOI: https://dx.doi.org/10.4314/jasem.v23i3.15}

Copyright: Copyright (C) 2019 Elekofehinti et al. This is an open access article distributed under the Creative Commons Attribution License (CCL), which permits unrestricted use, distribution, and reproduction in any medium, provided the original work is properly cited.

Dates: Received: 15 March 2019; Revised: 22 March 2019; Accepted 26 March 2019

Keywords: Insulin signaling, physicochemical characteristics, homology modelling, diabetes

Diabetes is a common metabolic disorder that affects about 422 million people globally (WHO, 2016; Elekofehinti, 2015). It is one of the leading causes of death worldwide and has been ranked as a major cause of blindness, kidney failure, heart attack, stroke and lower limb amputation (WHO, 2016). Obesity is a major risk factor for type 2 diabetes resulting from insulin resistance and is also linked to a number of other pathophysiological diseases such as atherosclerosis, hypertension and dyslipidemia (Elekofehinti et al, 2017; Kahn and Flier, 2000). Inositol hexakisphosphate kinase-1 (IP6K1) belongs to a family of inositol hexakisphosphate kinases (IP6Ks). IP6K1 has high-energy Beta phosphates that's readily phosphorylate proteins and modulate diverse cellular processes (Bhandari et al, 2008). IP6K1 has recently been linked to its insulin and glucose modulatory role (Bhandari et al, 2008; Kamimura et al, 2004). Ghoshal et al, (2016) reported that mice fed with high fat diet induced obesity and insulin resistance were protected through deletion of IP6K1 retreating the role of IP6K1 in energy homeostasis. Inositol pyrophosphates also known as, diphosphoinositol polyphosphates, are a family of water-soluble inositol phosphates. Diphosphoinositol pentakisphosphate (IP7) is the most notable due to its role in insulin secretion and peripheral insulin signaling (Mackenzie and Elliot, 2014; Barker et al, 2009; Werner et al, 2009). IP6K1 catalyzes the conversion of IP6 through phosphorylation to form inositol pyrophosphate, IP7. Production of IP7 results in its binding to protein kinase $\mathrm{B}(\mathrm{Akt} / \mathrm{PKB})$ thereby causing its inhibition and thus, prevents the translocation of glucose transporter 4 (Glut 4) to the cell membrane ultimately denying the cells (muscle and adipose tissue) of glucose uptake (Elekofehinti $e t$ al, 2017; Chakraborty et al, 2010). IP7 production by IP6K1 affects insulin-signaling pathway through 
potential inhibition of PDK1 phosphorylation of Akt which hamper insulin signaling cascade (Chakraborty et al, 2010; Sarbassov, 2005). Inhibition of IP6K1 is considered a novel approach in drug development towards type 2 diabetes and obesity. Ghoshal et al, (2016) recently identified [N2-(m-Triflurobenzyl), N6-(p-nitrobenzyl)purine] (TNP) as IP6K1 inhibitor that ameliorates diet induced obesity and insulin resistance making it a potential drug in the management of type 2 diabetes. In the present computational study, we employed various bioinformatics approach such as molecular docking and molecular dynamics simulation to predict the protein structure, understand its binding mode and dynamics.

\section{MATERIALS AND METHODS}

Structural modeling and validation: Amino acid sequence of IP6K1 was retrieved from GenBank database (accession no: AAH12944.1). IP6K1 modelling was done on SWISS-MODEL server.

Primary structure analysis of IP6K1: Expasy's Prot Param server (Gasteige et al, 2005) was used to study the physiochemical characters of IP6K1 and designed fusion constructs such as theoretical isoelectric point (pI), molecular weight, molecular formula, total number of positive and negative residues, instability index (Guruprasad et al, 1990), aliphatic index (Atsushi, 1980) and grand average hydropathicity (GRAVY) (Kyte and Doolittle, 1982). The instability index provides an estimate of a protein's stability in vitro. Proteins with instability index smaller than 40 are predicted as stable. A value above 40 indicates that the protein may be unstable.

Secondary structure analysis of IP6K1: Chou and Fasman Secondary Structure Prediction Server (CFSSP) was used to determine the secondary structure of IP6K1 in order to determine the helixes, sheets and turns (Chou and Fasman, 1974). The IP6K1 model was validated by ProSA server, Errat in SAVeS server (Laskowski et al, 1993). The phi/psi conformations were validated by the Ramachandran plot calculations using RAMPAGE software

\section{Molecular docking and scoring of TNP with IP6KI}

The mechanism of TNP binding was predicted by molecular docking analysis on IP6K1. The docking software Vina in PyMol plugin was used for better accuracy than AutoDock (Trott and Olson, 2010; DeLano, 2009). 3DLIGANDSITE and BSP-SLIM were employed to determine the best binding mode and binding pocket of TNP with IP6K1. A grid box size of $60 \times 60 \times 60$ with coordinates $\mathrm{x}=2.47, \mathrm{y}=-5.43$, $\mathrm{z}=20.29$ was built to cover the entire ligand binding site. The procedure was carried out by considering the flexibility of the ligand such that all rotational bonds were set free (Ji et al, 2017) and the estimated binding energies for the best pose was recorded.

Molecular Dynamics (MD) simulation: IP6K1: The MD simulation was performed using the program of GROMACS 4.6.5 MD package (Groingen Machine for Chemical Simulations) (Van Der Spoel et al., 2005) running on Mac OS $X$ (Processor $2.5 \mathrm{GHz}$ intel core i5 memory $4 \mathrm{~GB} 1600 \mathrm{MHz}$ DDR3) with GROMOS force field. Modeled IP6K1 was analyzed for MD approaches-molecular dynamics algorithm including an initial cubic solvation with single point charge water model, followed by ionization and neutralization in $\mathrm{Na}$ and $\mathrm{Cl}$ ions $(0.15 \mathrm{M})$. The solvated system was subjected to further energy minimization to remove stearic conflicts between protein and water molecules using the steepest descent integrator. The minimized model was subjected to position-restrained MD under NPT conditions by keeping the number of particles $(\mathrm{N})$, the system pressure $(\mathrm{P})$ and the temperature $(\mathrm{T})$ constant. This was executed for 100,000 steps for a total of $200 \mathrm{ps}$. The temperature of $300 \mathrm{k}$ and a pressure of $1 \mathrm{~atm}$ was maintained. After equilibration of the system with constant temperature and pressure, the production MD run of 10,000,000 steps was performed for $30 \mathrm{~ns}$ to carry out the structural analysis on IP6k1.

\section{RESULTS AND DISCUSSION}

Retrieval of human IP6K1 sequence (Accession No: AAH12944.1 GI: 15277917) was obtained from Genbank. The primary structure features of IP6K1 are presented in Table 1. The calculated isoeletric point (pI) for the protein is 6.81 suggesting that more positively charged residues are present within the protein molecule. There appears to be no disulphide bond as the presence of a disulphide bond increases the enthalpy of the folded state by stabilizing local interactions (Soni et al, 2013).

Table 1: Physico-chemical properties of IP6K1

\begin{tabular}{ll}
\hline Physico-ch emical param eters \\
\hline Molecular formula & $\mathrm{C}_{2171} \mathrm{H}_{3421} \mathrm{~N}_{639} \mathrm{O}_{681} \mathrm{~S}_{27}$ \\
Molecular weight & 50235.54 \\
p.I & 6.81 \\
$-\mathrm{R}$ (negative residue) & 62 \\
+R (positive residue) & 60 \\
Extinction coefficient & $34560 \mathrm{M}^{-1} \mathrm{~cm}^{-1}$ \\
Instability index & 50.53 \\
Aliphatic index & 65.83 \\
GRAVY & -0.724 \\
\hline
\end{tabular}

Though the protein has high aliphatic index (65.83), it is thermally unstable (with an instability index of 50.53) since an instability index of more than 40 
suggests instability. The Grand average hydropathy (GRAVY) value of IP6K1 (-0.724) suggests a hydrophilic pattern with better interaction with water. The sequence of IP6K1 was queried on Chou and Fasman secondary Structure Prediction server to determine the secondary structure. The sequence is made up of 441 amino acid residues categorized into helix, sheets and turns (Figure 1). The percentage of alpha helix, sheets and turns are $63.3 \%, 30.2 \%$ and $13.4 \%$ respectively.
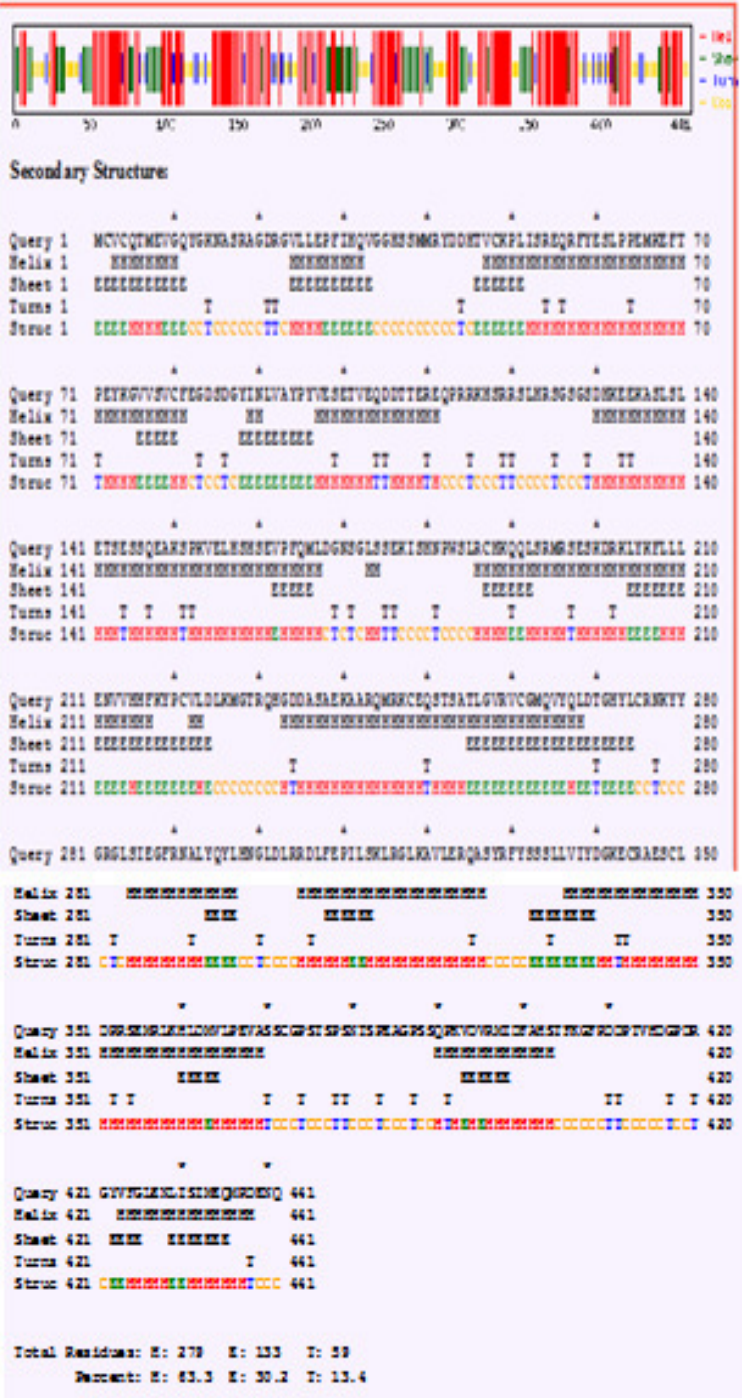

Fig 1: Secondary Structure predictions by CFSSP server

Three dimensional model is a tool needed to perform structural analysis on human IP6K1, hence, the 3D model of was constructed through homology modeling on SWISS-MODEL web server (Figure 2).

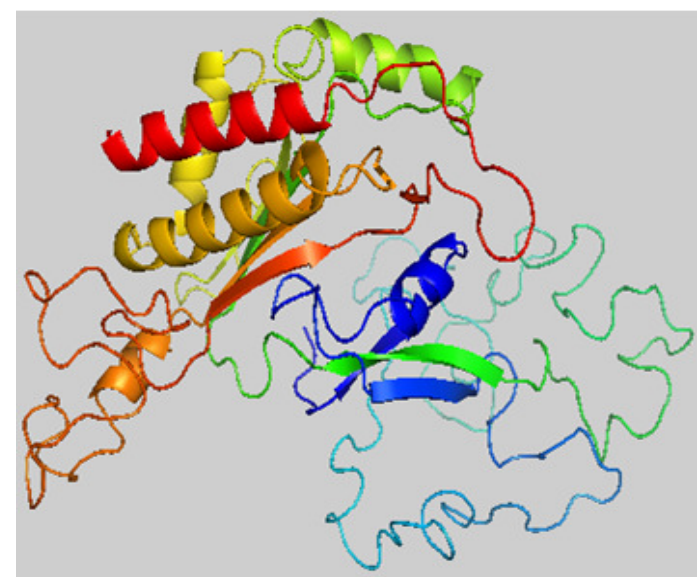

Fig 2. Three dimensional structure (3D) of the human IP6K1 generated by SWISS-MODEL server.

The overall quality of the protein model was validated using ProSA server (Wiederstein and Sippl, 2007) with the quality index represented by a Z-score of 5.36 (Figure 3) which is not too different from that of experimental protein structure (Figure 4). Ramachandran plot was also used to validate the model as described by Lovell et al, 2002 (Figure 5).

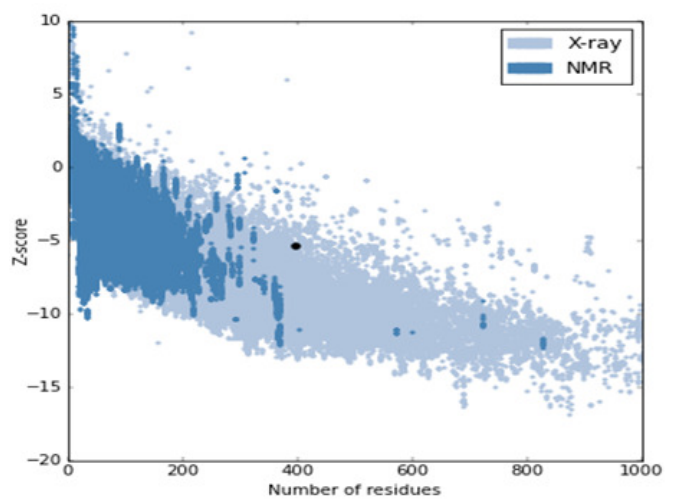

Fig 3. Quality index of the IP6K1 protein model generated by ProSA server. The Z-score (-5.36) indicates the local model quality.

In order to investigate the binding pocket and critical residues involved in IP6K1, we used AurodockVina to dock TNP into the predicted binding pocket "active site" of IP6K1 using 3DLIGANDSITE and BSPSLIM servers (Figure 4). The affinity of TNP to IP6K1 is $-8.1 \mathrm{kcal} / \mathrm{mol}$ (Table 2). Figure 7 showed the binding mode of the ligand within the predicted active site. The ligand TNP interacted with the residues within the active site through hydrogen bonding and hydrophobic interaction. TNP formed three hydrogen bonds with Tyr 59, Asn 212 and ASP 399, while Arg 41, Tyr 42, Val 47, Lys 49, Pro 71, Leu 210, Glu 211, Val 213, Ile 398 and Leu 336, formed hydrophobic interaction with TNP (Table 2). 


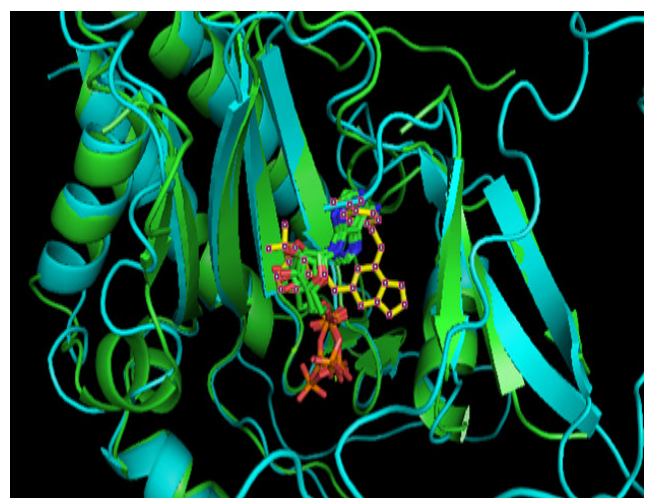

Fig 4: Projection of the predicted active site for IP6K1 with 3dligandsite (green) and BSP-SLIM (cyan) RMS = 1.697

Table 2. Molecular docking results of human IP6K1 with TNP Binding affinity Hydrogen bond Hydrophobic interaction $\left(\mathrm{kcalmol}^{-1}\right)$

\begin{tabular}{|c|c|c|}
\hline TNP -8.1 & $\begin{array}{l}3 \text { (Tyr 59, Asn } 212 \\
\text { Asp 399) }\end{array}$ & $\begin{array}{l}10 \text { (Arg 41, Tyr 42, Val 47, } \\
\text { Lys 49, Pro 71, Leu 210, } \\
\text { Glu 211, Val213, Ile } 398 \\
\text { and Leu 336) }\end{array}$ \\
\hline
\end{tabular}

Assessment of protein quality is imperative in structural proteomics. The overall protein structure as well as function is a factor of the amino acid sequence that represents the primary structure of protein. The sequence of amino acid of the protein having 441 residue was retrieved from Genbank. The sequence was used to perform homology modelling on SWISSMODEL server. The modelled protein is a hypothetical one with a molecular weight of approximately 50235.54 Da. Aliphatic index value is a function of structural stability of any protein indicating the relative volume occupied by amino acids having aliphatic side chains in their structure such as leucine, valine, isoleucine and alanine. It is a positive factor for the increase of thermostability of globular proteins (Ikai, 1980; Bhattacharjee et al, 2016). The estimated aliphatic index for IP6K1 was 65.83. Instability index gives estimate information about the stability of the protein and the score for IP6K1 in this study was 50.53 and could be considered as unstable. The instability index provides an estimate of the stability of any protein. The estimated value for IP6K1 is 50.53 indicating that the protein model may be unstable since protein with instability index is smaller than 40 is predicted as stable a value above 40 predicts that the protein may be unstable (Guruprasad et al, 1990). Protein $\mathrm{pI}$ is calculated using $\mathrm{pKa}$ values of amino acids. The pKa value of amino acids depends on its side chain. It has an important role in defining the $\mathrm{pH}$ dependent characteristics of a protein and can be used to optimize IP6K1 purification and crystallization. The estimated pI for IP6K1 was 6.81 showing a nearly neutral nature of the protein. In the protein molecule, serine has the highest composition with $11.3 \%, 9.1 \%$ Leucine, $8.2 \%$ of glutamic acid, $7.9 \%$ arginine while the lowest was tryptophan with $0.2 \%$. The total number of negatively charged residues $($ Asp + Glu $)$ is 62 while that of positively charged residues $(\mathrm{Arg}+\mathrm{His})$ are 60 (Table 1). The atomic composition of the protein indicated that it has 6393 atoms consisting of 2171 carbon, 3421 hydrogen, 639 nitrogen, 681 oxygen and 27 surphur and has the formula $\mathrm{C}_{2171} \mathrm{H}_{3421} \mathrm{~N}_{639} \mathrm{O}_{681} \mathrm{~S}_{27}$. The degree of hydrophobicity or hydrophilicity of a protein is measured by the Grand average of hydropathicity (GRAVY) and it is an important parameter during protein characterization. The calculated score was 0.724 showing that the protein is more globular and hydrophilic (Table 1). The secondary structure prediction was done using the primary amino acid sequence through CFFSP webserver. The modeled IP6K1 has $63.3 \%$ helixes, $30.2 \%$ sheets and $13.4 \%$ turns (Figure 1). Validation of the model is very important in protein structural prediction since ultimately the modeled protein structure is used to understand the protein's biological function in order to design further experiments. The quality of the model was assessed using the ProSA server (Figure 3) and validated by RAMPAGE A Ramachandran plot was obtained from RAMPAGE for the generated $\mathrm{pdb}$ structures of IP6K1 (Figure 5). The Ramachandran plot from RAMPAGE showed that the model contains $78.1 \%$ including 310 residues in the most favoured region.

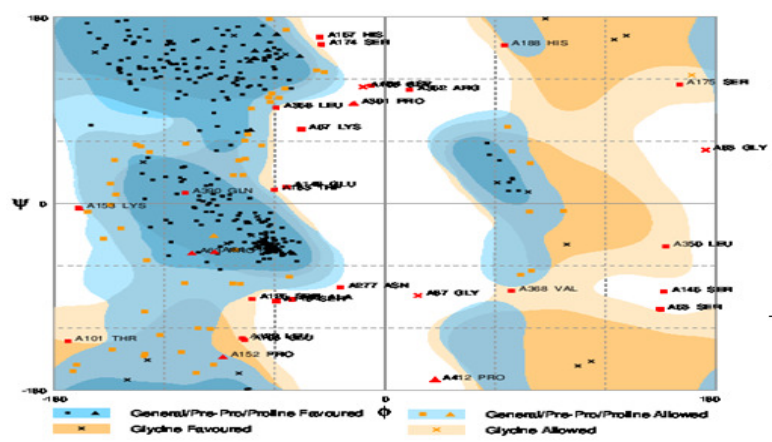

Fig 5: Ramachandran plot of IP6K1 protein model. The most favored regions, additional allowed regions, generously allowed regions, and disallowed regions are indicated as dark blue, light blue, light yellow, and white, respectively.

The primary sequence of IP6K1 was used to model the protein using SWISS-MODEL server (Figure 3) though homology modelling with protein 21 ew. 2 serving as template. The binding pocket of the protein was determined using two webservers; 3ligandsite and BSP-SLIM. The binding pocket identified by the two webservers were found to be the same as shown in Figure 4. In order to investigate the binding mode and 
critical residues involved in IP6K1, molecular docking was performed with TNP using AutodockVina. The results showed top ten binding conformations of TNP with IP6K1. Among the ten conformations, the best with a least binding energy $-8.1 \mathrm{kcalmol}^{-1}$ was selected. Weak intermolecular interactions such as hydrogen bonding and hydrophobic interactions are key players in stabilizing energetically-favored ligands, in an open conformational environment of protein structures (Patil et al, 2010). Three hydrogen bonding interactions was observed between IP6K1 and TNP (Table 2). The binding mode and binding cavity of TNP with IP6K1 was visualized using PyMol (Figure 6).

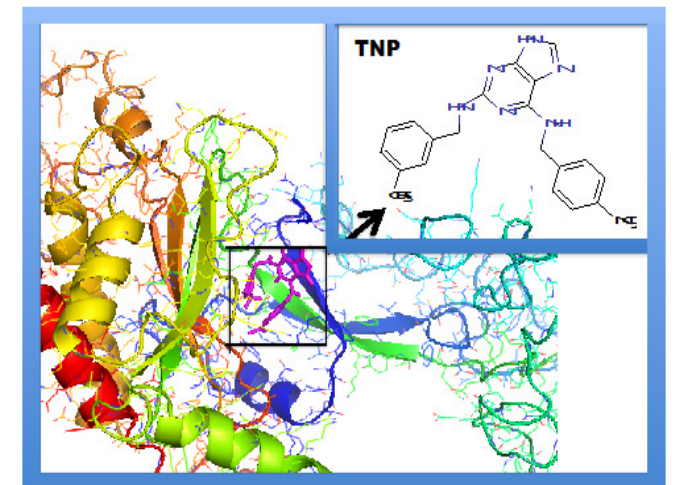

Fig 6. Binding pose of IP6K1 with TNP with binding affinity of $8.1 \mathrm{kcal} / \mathrm{mol}$

The residues Tyr59 Asn212 and Asp399 formed three hydrogen bonds with TNP and identified as critical residues in TNP binding. In addition, hydrophobic and hydrogen bonding interactions were predicted for the IP6K1-TNP complex using the Ligplot tool. The results showed that residues Arg41, Tyr42, Val47, Lys49, Pro71, Leu210, Glu211, Val213, Ile398 and Leu336 were involved in hydrophobic interactions with TNP atoms (Figure 7).

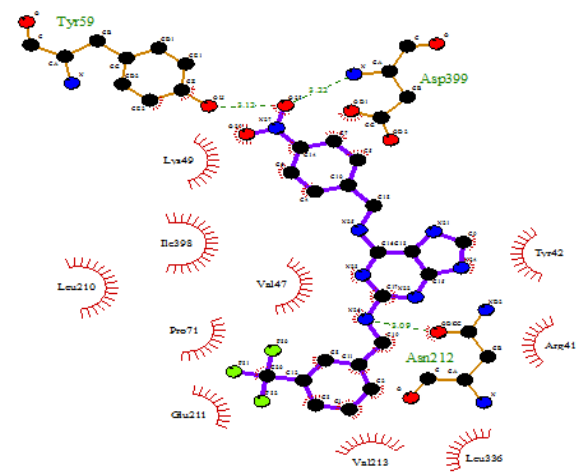

Fig 7. Molecular docking of IP6K1 with TNP showing hydrogen bonding and hydrophobic interactions

Molecular dynamics (MD) simulation of modelled IP6K1 was carried out using the Molecular Dynamics simulation software (GROMACS) package. The structural variations during the course of the simulations were analyzed by calculating RMSD, RMSF, radius of gyration ( $\mathrm{Rg}$ ) and solvent accessible surface area (SAS). Protein simulation is a highly complex process that provides information about the physical movement and interaction of atoms and molecules revealing a whole new insight into protein dynamics (Yang et al, 2013). In the current study, Root mean square deviation (RMSD) provides information about a protein with respect to its backbone structure. An initial large fluctuation was observed (Figure 8) which was stabilized after $10 \mathrm{~ns}$ due to reduction in amplitude of fluctuation with time. The Root mean square fluctuation (RMSF) gives information about the dynamic behavior of residues. The result revealed large fluctuation between 100 and 200 and between 350 and 400 (Figure 9).

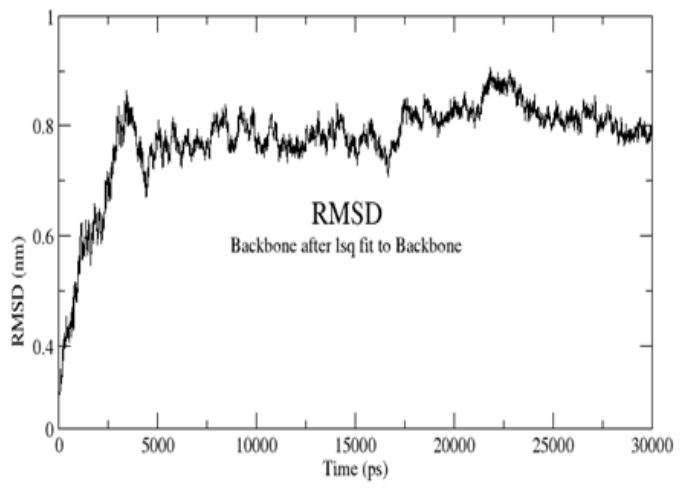

Fig 8. RMSD plot of backbone atoms of IP6K1 in apo state

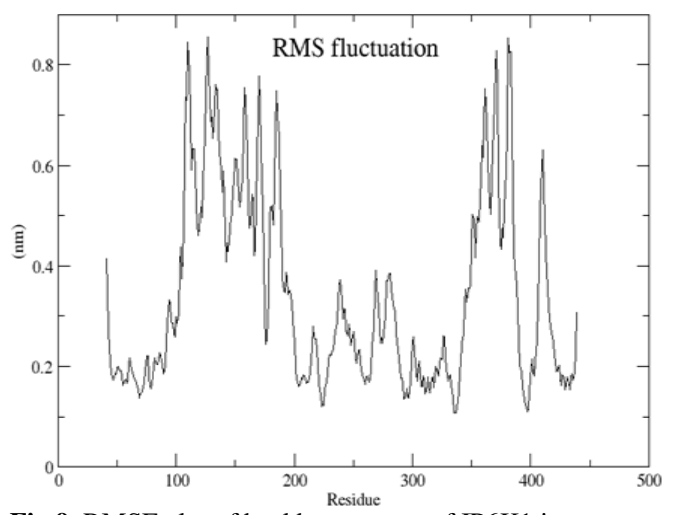

Fig 9. RMSF plot of backbone atoms of IP6K1 in apo state

This fluctuation could be due to the presence of loops within the protein molecule. The radius of gyration indicates the degree of compactness of a molecule. The result from this study showed the protein in folding and unfolding configuration and the protein appeared to be stable around 20ns (Figure 10). 


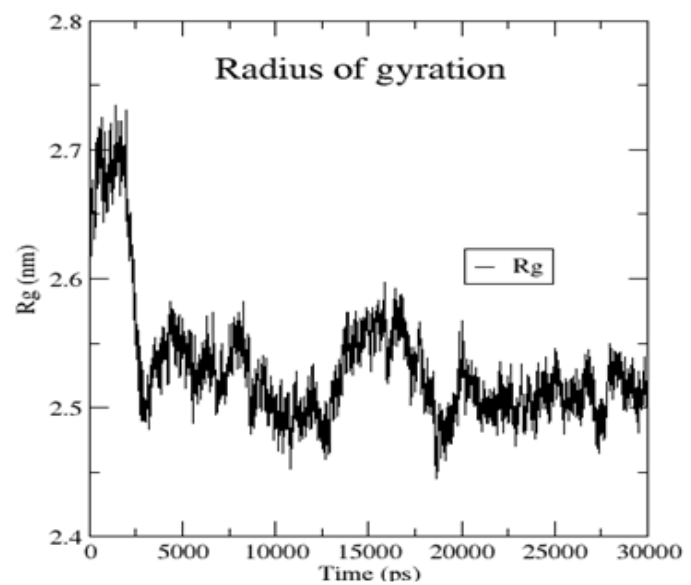

Fig 10. Radius of gyration $(\mathrm{Rg})$ plot of IP6K1 in apo state

The solvent accessible surface (SASA) analysis was carried out to explore the behavior of hydrophilic SASA and hydrophobic SASA of the IP6K1. SASA results (Figure11) of around 125-130 nm2 showed that the accessibility was retained with little changes during the simulation and confirmed that the residues were well exposed to the solvent (Lin et al, 2003).

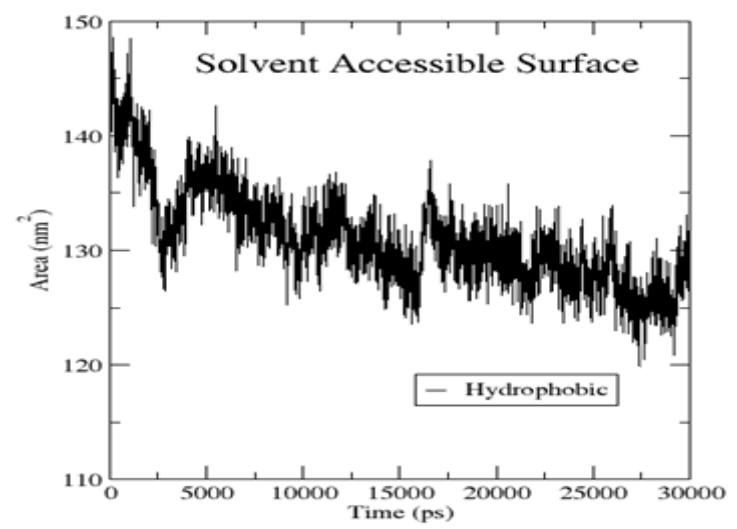

Fig 11. The solvent accessible surface (SASA) plot of IP6K1 in apo state

This study provides insight into dynamics of IP6K1 as this data will assist in the conformational studies, enzyme reactions and molecular signaling between IP6K1 and ligands.

Conclusion: Human IP6K1 plays a critical role in insulin signaling which is one of the key mechanisms in diabetes treatment. Experimental studies supported IP6K1 as a novel therapeutic target for diabetes. IP6K1 structure has been predicted the model was stable. Tyr59, Asn212 and Asp399 were found to be critical residues in ligand binding. Our computational studies reavealed the binding pocket and critical amino acid residues that can be exploited in the design of inhibitors of this enzymes as antidiabetic drugs.

\section{REFERENCES}

Barker, CJ, Illies, C, Gaboardi, GC, Berggren, PO (2009). Inositol pyrophosphates: structure, enzymology and function. Cellular Mol. Life Sci. 66: 3851

Bhandari, R, Juluri, KR, Resnick, AC, Snyder, SH (2008). Gene deletion of inositol hexakisphosphate kinase 1 reveals inositol pyrophosphate regulation of insulin secretion, growth, and spermiogenesis. Pro. Natl. Acad. Sci. 105: 2349-2353

Bhattacharjee, B, Pathaw, N, Chrungoo, NK, Bhattacharjee, A (2017). Molecular modelling, dynamics simulation and characterization of antifungal chitinase from Sechium edule. Gene 606: $39-46$

Chakraborty, A, Koldobskiy, MA, Bello, NT, Maxwell, M, Potter, JJ, Juluri, KR, Saleh, M (2010). Inositol pyrophosphates inhibit Akt signaling, thereby regulating insulin sensitivity and weight gain. Cell 143: 897-910.

Chou, PY, Fasman, GD (1974). Conformational parameters for amino acids in helical, $\beta$-sheet, and random coil regions calculated from proteins. Biochem. 13: 211-222

DeLano, WL (2017). The PyMOL Molecular Graphics System. Available online: http://pymol.sourceforge.net/overview/index.htm (accessed on 13 May 2017)

Elekofehinti, OO (2015). Saponins: Anti-diabetic principles from medicinal plants-A review. Pathophysiol. 22: 95-103.

Elekofehinti, OO, Ejelonu, OC, Kamdem, JP, Akinlosotu, OB, Adanlawo, IG (2017). Saponins as adipokines modulator: A possible therapeutic intervention for type 2 diabetes. W. J. Diabetes 8: 337

Gasteiger, E, Hoogland, C, Gattiker, A, Wilkins, M, Appel, RD, Bairoch, A (2005). Protein identification and analysis tools on the ExPASy server. In The proteomics protocols handbook Humana press 571-607

Ghoshal, S, Zhu, Q, Asteian, A, Lin, H, Xu, H, Ernst, G, Chakraborty, A (2016). TNP [N2-(mTrifluorobenzyl), N6-(p-nitrobenzyl) purine] ameliorates diet induced obesity and insulin resistance via inhibition of the IP6K1 pathway. Mol. Metabol. 5: 903-917 
Guruprasad, K, Reddy, BB, Pandit, MW (1990). Correlation between stability of a protein and its dipeptide composition: a novel approach for predicting in vivo stability of a protein from its primary sequence. Protein Eng., Design Selec. 4: $155-161$.

Ikai, A (1980). Thermostability and aliphatic index of globular proteins. J. Biochem. 88: 1895-1898

Ji, X, Xiao, Y, Liu, S (2018). Structural modeling of human cardiac sodium channel pore domain. J. Biomole. Struct. Dynam. 36: 2268-2278.

Kahn, BB; Flier, JS (2000). Obesity and insulin resistance. J. Clin. Invest. 106: 473-481

Kamimura, J, Wakui, K, Kadowaki, H, Watanabe, Y, Miyake, K, Harada, N, Kishino, T (2004). The IHPK1 gene is disrupted at the 3p21. 31 breakpoint of $\mathrm{t}(3 ; 9)$ in a family with type 2 diabetes mellitus. J. Hum. Genet. 49: 360

Kyte, J, Doolittle, RF (1982). A simple method for displaying the hydropathic character of a protein. J. Mol. Bio. 157: 105-132

Laskowski, RA, MacArthur, MW, Moss, DS, Thornton, JM (1993). PROCHECK: a program to check the stereochemical quality of protein structures. J. App. Crystallo. 26: 283-291

Lin, L, Thomas, A, Brasseur, R (2003). Analysis of accessible surface of residues in proteins. Protein Sci. 12: 1406-1417.

Lovell, SC, Davis, IW, Arendall, WB, De Bakker, PI, Word, JM, Prisant, MG, Richardson, DC (2003). Structure validation by $\mathrm{C} \alpha$ geometry: $\phi, \psi$ and $\mathrm{C} \beta$ deviation. Proteins: Stru. Func. Bioinform. 50: 437-450

Mackenzie, RW, Elliott, BT (2014). Akt/PKB activation and insulin signaling: a novel insulin signaling pathway in the treatment of type 2 diabetes. Diabetes, metabolic syndrome and obesity: Targets Ther. 7: 55
Patil, R, Das, S, Stanley, A, Yadav, L, Sudhakar, A, Vama, A (2010). Optimized hydrophobic interactions and hydrogen bonding at the target ligand interface leads the pathways of drugdesigning. Plus One 5: e12029

Sarbassov, DD, Guertin, DA, Ali, SM, Sabatini, DM (2005). Phosphorylation and regulation of Akt/PKB by the rictor-mTOR complex. Sci. 307: 1098-1101.

Soni, SP, Adu-Gyamfi, E, Yong, SS, Jee, CS, Stahelin, RV (2013). The Ebola virus matrix protein deeply penetrates the plasma membrane: an important step in viral egress. Biophy. J. 104: 1940-1949

Trott, O, Olson, AJ (2010). AutoDock Vina: improving the speed and accuracy of docking with a new scoring function, efficient optimization, and multithreading. J. Comput. Chem. 31: 455-461

Van Der Spoel, D, Lindahl, E, Hess, B, Groenhof, G, Mark, AE, Berendsen, HJ (2005). GROMACS: fast, flexible, and free. J. Comput. Chem. 26: $1701-1718$

Werner, JK, Speed, T, Bhandari, R (2010). Protein Pyrophosphorylation by Diphosphoinositol Pentakisphosphate (InsP 7). In Inositol Phosphates and Lipids Humana Press 87-102

WHO 2016. Global report on diabetes. World Health Organization, Geneva, 2016.

Wiederstein, M, Sippl, MJ (2007). ProSA-web: interactive web service for the recognition of errors in three-dimensional structures of proteins. Nucl. Acids Res. 35: 407-410.

Yang, L, Sang, P, Tao, Y, Fu, Y, Zhang, K, Xie, Y (2013). Protein dynamics and motions in relation to their functions: several case studies and the underlying mechanisms. J. Biomol. Stru. Dynam. 32: 372-393. 